\title{
Decision models of Multi Periods Closed Loop Supply Chain with Remanufacturing under Centralized and Decentralized Decision Making
}

\author{
JiangJun Yuan \\ Department of Shangmao, Zhejiang Technical Institute of Economics, Hangzhou, \\ Zhejiang, 310018, China \\ E-mail:Welleb@qq.com
}

\begin{abstract}
The closed-loop supply chains for new products and remanufactured products under uncertain demand are studied. Considering the differences in consumers' "willingness to pay (WTP)" for new products and remanufactured products, the decision models of multi-period closed-up supply chain with remanufacturing under centralized and decentralized decision making are established respectively and optimal decision of each member of the supply chain is obtained. Through comparison between differences in the consumers' WTP for the two types of products, three closed-loop supply chains are established under the condition of the government's reward and punishment mechanism. The game model and numerical experiment dominated by various manufacturers are elaborately analyzed, the interest coordination mechanism is confirmed with Shapley Value Method, and the model is solved and dissected through numerical simulation. The research results show there is benefit loss under decentralized decision making, and a reduction of middlemen can effectively improve benefits of the supply chain. The benefits of closed-loop supply chain with remanufacturing can be distributed with Shapley Value in a rational and effective manner.
\end{abstract}

Keywords: Closed-loop supply chain, WTP Differentiation, Closed-loop supply chain, Remanufacturing, Reward and punishment mechanism

\section{Introduction}

Currently, as the contradiction between global resources and environment increasingly intensified, countries in the world put more emphasis on sustainable development and circular economy, and have passed laws successively to enhance and extend the awareness and responsibility for recycling waste products [1]. The operation mode of closed-loop supply chain incorporating traditional forward supply chain and reverse supply chain has become the research hotspot in business circles and academic circles. Decision problems of the traditional supply chain belong to the decentralized decision making. Each enterprise makes the best decisions given the available information without achieving Pareto optimal state. Mutual cooperation among enterprises is needed to realize profit maximization of the entire closed-loop supply chain system, and a reasonable and effective interest coordination mechanism will exert direct influence on the operation efficiency of closed-loop supply chain under centralized decision-making. Savaskan conducted a game analysis on problem of the optimal channel structure [1]. Wang Yuyan studied the closed-loop supply chain comprised of a single manufacture and an exclusive retailer, and presented a profit share mechanism distributed by proportion of the system gain [2]. Later on, Wang Yuyan researched the coordination of closed-loop supply chain aimed at recycling mode of the third party - recyclable waste collector, which showed that a greater cooperation among insiders of the closed-loop supply chain can bring more profits through the channel [3]. Sheng Fangzheng discussed the rational benefit allocation 
method of two-echelon supply chain including a supplier and several retailers [4]. Wang $\mathrm{Li}$ constructed the "multi-directional and principal-subordinate mode" three-echelon supply chain and quantitatively analyzed the downstream cooperation profit allocation mechanism [5]. Zhang Yun built the profit allocation model between the general contractor and the subcontractor in view of revenue sharing theory and Stackelberg Game [6].

The above-mentioned documents have deeply researched the interest coordination of closed-loop supply chain, but without taking account of remanufacturing. Therefore, some scholars discussed the interest coordination mechanism of closed-loop supply chain with remanufacturing. Bao Xiaoying proposed the two-part tariff contract to coordinate pricing strategies and realized the coordination of closed-loop supply chain with remanufacturing [7]. Zhou balanced the supply and demand of closed-loop supply chain with remanufacturing by using the dynamic pricing strategy [8]. Wei Jie studied the pricing and coordination problems of closed-loop supply chain with manufacturing/ remanufacturing in the vague and uncertain context [9]. The remanufactured products and new products above are together sold to the consumers, failing to take the differences between these two types of products into consideration. However, study from Guide VDR indicated that there existed significant difference in the consumers' "willingness to pay" for new products and remanufactured products, which would create a remarkable circle of influence on the manufacturers' decision-making and strikingly affect each decision maker of the closed-loop supply chain.

\section{Model Assumption}

To more clearly illustrate the model, the assumption below is made:

(1). Consider only a single manufacturer, a single distributor and an exclusive retailer who are all risk neutral and of perfect information. The manufacturer is the Stackelberg leader, and the distributor is a follower relative to the manufacturer while a leader relative to the retailer.

(2). Suppose variable cost per unit of the new products and remanufactured products are $c_{n}$ and $c_{r}$ respectively and $c_{n}>c_{r}>0$, which reflects cost saving benefits of the remanufactured products.

(3). According to the conclusions of document [12], there exist differences in the consumers' WTP for new products and remanufactured products. Provided consumers' WTP for these two types of products are 1 and $\beta$

rspectively, and the client utility is uniformly distributed in $[0,1]$, the law of substitution the demand function fits is : $p_{n}=1-q_{n}-\beta q_{r}, p_{r}=\beta\left(1-q_{n}-q_{r}\right)$

Wherein $0 \leq \beta \leq 1$ refers to the consumers' WIP for new products are higher than that for remanufactured products. Because only the new products are produced in the first period, and the new products and remanufactured products are together produced in the second period, the demand function below is created:

$$
\begin{aligned}
& p_{1 n}=1-q_{1 n}, \\
& p_{2 n}=1-q_{2 n}-\beta q_{r}, \\
& p_{r}=\beta\left(1-q_{2 n}-q_{r}\right) ;
\end{aligned}
$$

(4) Suppose recycling rate for EOL products is $\theta(0 \leq \theta \leq 1)$, all the recycled products are intended for remanufacturing with no loss. Fixed investment from manufacturers for EOL products is $I(\theta)=B \theta^{2}$ [14] with the scale parameter $B>0$.

(5) There being no consideration of the government subsidiaries etc., in this paper, suppose $A<c_{n}-c_{r}$, that is to say, the recycling cost from manufacturers for EOL products is lower than the remanufacturing profit, which brings them an incentive to recycle the EOL products.

(6) Suppose the recycled EOL products can meet the manufacturers' requirements for 
producing remanufacturing products.

\section{Model Design}

\subsection{Decision-making Analysis under Decentralized Decision Making}

Given the assumptions above, pursuit for profit maximization is set as the decision goal for members of the supply chain, and the manufacturer, the distributer and the retailer constitute a three-stage Stackelberg Game. First of all, the retailer confirms the optimal retail price of new products and remanufactured products are $p_{1 n}, p_{2 n}$ and $p_{r}$ in the light of the distributor's wholesale price $w_{1 D}, w_{2 D}$ and $w_{r D}$. Then, the distributor determines the wholesale price depending on order quantity of the retailer and ex-factory price of the manufacturer. Finally, the manufacturer makes the best ex-factory price $w_{1 S}$, $w_{2 S}$ and $w_{r S}$ according to cost of production and order quantity.

On account that the retailer merely markets the new products and remanufacturing products, the optimal decision problem of gross profit $\pi_{R}^{D}$ for the retailer within two periods is:

$$
\begin{aligned}
& \max _{p_{1 n}, p_{2 n}, p_{r}} \pi_{R}^{D}=\left(p_{1 n}-w_{1 D}\right) q_{1 n} \\
& +\left(p_{2 n}-w_{2 D}\right) q_{2 n}+\left(p_{r}-w_{r D}\right) q_{r}
\end{aligned}
$$

Plug the demand function in assumption 3 into the above equation:

$$
\begin{aligned}
& \max _{p_{1 n}, p_{2 n}, p_{r}} \pi_{R}^{D}=\left(p_{1 n}-w_{1 D}\right)\left(1-p_{1 n}\right)+ \\
& \left(p_{2 n}-w_{2 D}\right)\left(1-\frac{p_{2 n}-p_{r}}{1-\beta}\right)+\left(p_{r}-w_{r D}\right) \frac{p_{r}-\beta p_{2 n}}{\beta(\beta-1)}
\end{aligned}
$$

Evaluate the first-order partial derivatives of $p_{1 n}, p_{2 n}$ and $p_{r}$ in equation (1), make them equal to zero, and get response function of the retailer about the wholesale price $w_{1 D}, w_{2 D}$ and $w_{r D}$ :

$$
p_{1 n}=\frac{1+w_{1 D}}{2}, p_{2 n}=\frac{1+w_{2 D}}{2}, p_{r}=\frac{\beta+w_{r D}}{2}
$$

Later on, the distributor sets the best wholesale price of products in line with the retailer's decision-making and the gross profit $\pi_{D}^{D}$ can be indicated by:

$$
\begin{aligned}
& \max _{w_{1 D}, w_{2 D}, w_{r D}} \pi_{D}^{D}=\left(w_{1 D}-w_{1 S}\right) q_{1 n}+ \\
& \left(w_{2 D}-w_{2 s}\right) q_{2 n}+\left(w_{r D}-w_{r S}\right) q_{r}
\end{aligned}
$$

Substitute the demand function and the retailer's response function in assumption 3 into equation (2), evaluate the first-order partial derivatives of $w_{1 D}, w_{2 D}$ and $w_{r D}$, make them equal to zero, and get the response function of distributor about the ex-factory price $w_{1 D}, w_{2 D}$ and $w_{r D}$ :

$$
w_{1 D}=\frac{1+w_{1 S}}{2}, w_{2 D}=\frac{1+w_{2 S}}{2}, w_{r D}=\frac{\beta+w_{r S}}{2}
$$

At last, the manufacturer recycles and remanufactures the new products and EOL products on the basis of the distributor's decision-making, and the gross profits $\pi_{m}^{D}$ can be indicated by:

$$
\begin{aligned}
& \max _{w_{1 S}, w_{2 S}, w_{r S}, \theta}^{D}=\left(w_{1 S}-c_{n}\right) q_{1 n}+\left(w_{2 S}-c_{n}\right) q_{2 n} \\
&+\left(w_{r S}-c_{r}\right) q_{r}-B \theta^{2}-A \theta q_{1 n}
\end{aligned}
$$

Similarly, plug the demand function and the distributor's response function in assumption 3 into equation (3), evaluate the first-order partial derivatives of $w_{1 S}, w_{2 S}$, $w_{r S}$ and $\theta$, make them equal to zero, then: 


$$
\begin{aligned}
& w_{1 S}^{D *}=\frac{A^{2}-8 B\left(1+c_{n}\right)}{A^{2}-16 B}, w_{2 S}^{D *}=\frac{1+c_{n}}{2}, \\
& w_{r S}^{D^{*}}=\frac{\beta+c_{r}}{2}, \theta^{D^{*}}=\frac{\left(1-c_{n}\right) A}{A^{2}-16 B}
\end{aligned}
$$

Substitute the above equation into the distributor's response function, then:

$w_{1 D}^{D *}=\frac{1}{2}+\frac{A^{2}-8 B\left(1+c_{n}\right)}{2\left(A^{2}-16 B\right)}, w_{2 D}^{D *}=\frac{3+c_{n}}{4}, w_{r D}^{D *}=\frac{3 \beta+c_{r}}{4}$

Similarly, plug the above equation into the retailer's response equation, then:

$$
p_{1 n}=\frac{3}{4}+\frac{A^{2}-8 B\left(1+c_{n}\right)}{4\left(A^{2}-16 B\right)}, p_{2 n}=\frac{7+c_{n}}{8}, p_{r}=\frac{7 \beta+c_{r}}{8}
$$

Profits of the manufacturer, the distributor, the retailer and the entire closed-loop supply chain with remanufacturing system can be further obtained:

$$
\begin{aligned}
& \pi_{M}^{D^{*}}=\frac{\left(\beta c_{n}-c_{r}\right)^{2}}{16 \beta(1-\beta)}+\frac{\left(1-c_{n}\right)^{2}}{16}-\frac{B\left(1-c_{n}\right)^{2}}{A^{2}-16 B}, \\
& \pi_{D}^{D^{*}}=\frac{\left(\beta c_{n}-c_{r}\right)^{2}}{32 \beta(1-\beta)}+\frac{\left(1-c_{n}\right)^{2}}{32}+\frac{8 B^{2}\left(1-c_{n}\right)^{2}}{\left(A^{2}-16 B\right)^{2}}, \\
& \pi_{R}^{D^{*}}=\frac{\left(\beta c_{n}-c_{r}\right)^{2}}{64 \beta(1-\beta)}+\frac{\left(1-c_{n}\right)^{2}}{64}+\frac{4 B^{2}\left(1-c_{n}\right)^{2}}{\left(A^{2}-16 B\right)^{2}}, \\
& \pi_{T}^{D^{*}}=\frac{7\left(\beta c_{n}-c_{r}\right)^{2}}{64 \beta(1-\beta)}+\frac{7\left(1-c_{n}\right)^{2}}{64} \\
& -\frac{\left(A^{2}-28 B\right) B\left(1-c_{n}\right)^{2}}{\left(A^{2}-16 B\right)^{2}} ;
\end{aligned}
$$

\subsection{Decision-making under Centralized Decision Making}

Under decentralized decision making, members of the supply chain solely make an effort to maximize their profits, in which case the whole supply chain may not achieve the profit maximization. Under centralized decision making (model $\mathrm{C}$ ), the manufacturer, the distributor and the retailer will make decisions as a whole to fulfill the decision goal of maximizing total profits of the remanufacturing closed-loop supply chain. Under this circumstance, the decision problem can be indicated by:

$$
\begin{aligned}
& \underset{p_{1 n}, p_{2 n}, p_{r}, \theta}{\max }=\left(p_{1 n}-c_{n}\right) q_{1 n}+\left(p_{2 n}-c_{n}\right) q_{2 n} \\
& +\left(p_{r}-c_{r}\right) q_{r}-B \theta^{2}-A \theta q_{1 n}
\end{aligned}
$$

Plug the demand function in assumption 3 into equation (5), evaluate the first-order partial derivatives of $p_{1 n}, p_{2 n}, p_{2 n}$ and $w_{r D}$, and make them equal to zero, then:

$$
\begin{aligned}
& p_{1 n}^{C^{*}}=\frac{A^{2}-2 B\left(1+c_{n}\right)}{A^{2}-4 B}, p_{2 n}^{C^{*}}=\frac{1+c_{n}}{2}, \\
& p_{r}^{C^{*}}=\frac{\beta+c_{r}}{2}, \theta^{C^{*}}=\frac{A\left(1-c_{n}\right)}{A^{2}-4 B}
\end{aligned}
$$

Substitute the equation above into equation (5) and acquire profits of the entire closed-loop supply chain with remanufacturing:

$$
\pi_{T}^{C *}=\frac{\left(\beta c_{n}-c_{r}\right)^{2}}{4 \beta(1-\beta)}+\frac{\left(1-c_{n}\right)^{2}}{4}-\frac{B\left(1-c_{n}\right)^{2}}{A^{2}-4 B},
$$




\subsection{Interest Coordination Mechanism}

Under centralized decision making, a reasonable and effective interest coordination mechanism is required to please each member of the supply chain. Because Shapley Value Method must be of the unique solution and is easy to be quantitatively calculated, it is most widely used in game theory, economics and other social studies. Profits of the closed-loop supply chain with remanufacturing will be allocated rationally with Shapley Value Method in this paper.

Suppose the cooperative game $\eta=(N, v)$ wherein $N=\{1,2 \cdots, n\}$ refers to a set of participants and $\mathrm{n}$ is an integer referring to number of participants. $\mathrm{S}$ is the subset of $\mathrm{N}$ and refers to the alliance of participants, i.e. $S \subseteq N$. v is an eigenfunction each alliance $\mathrm{S}$ in $\mathrm{N}$ wants to correspond; $v(S)$ refers to the obtainable utility when the members in alliance $\mathrm{S}$ work together; $v(S / i)$ refers to the gettable utility when the member $\mathrm{i}$ is removed under the cooperation S. Shapley Values for obtainable utility of each participant under the cooperation $\mathrm{N}$ are:

$$
\psi_{i}(v)=\sum_{S \subseteq N, i \subseteq S} \gamma_{n}(s)(v(S)-v(S / i)), \quad i=1,2 \cdots n
$$

Wherein $\gamma_{n}(s)=((s-1) !(n-s) !) / n !, \psi_{i}(v)$ refers to the gettable utility of member i under the cooperation $\mathrm{N}$.

$n=3$ in the game model in this paper. The alliances the manufacturers participate in are $\{M\},\{M, D\},\{M, D, R\} \quad$; the alliances the distributors participate in are $\{D\},\{M, D\},\{D, R\},\{M, D, R\}$; the alliances the retailers participate in are $\{R\},\{D, R\},\{M, D, R\}$. On account that manufacturer $\mathrm{M}$ cannot form an alliance with retailer $\mathrm{R}, v(\{M, R\})$ are the total profits of manufacturer $\mathrm{M}$ and retailer $\mathrm{R}$ under decentralized decision-making. Centralized and decentralized decision-making have been discussed above and now it comes to the analysis on decision-making when the manufacturer and the distributor ally, and the distributor and the retailer ally.

3.3.1 Alliance of the Manufacturer and the Distributor: In this case, the retailer decides optimum retail price and order quantity of the new products and the remanufactured products, and then the manufacturer and distributor ascertain best wholesale price of the products with centralized decision-making. Respective profits of the retailer and the alliance MD are:

$$
\begin{aligned}
& \max _{p_{1 n}, p_{2 n}, p_{r}} \pi_{R}^{A_{1}}=\left(p_{1 n}-w_{1 S}\right) q_{1 n}+ \\
& \left(p_{2 n}-w_{2 S}\right) q_{2 n}+\left(p_{r}-w_{r S}\right) q_{r} \\
& \quad \max \pi_{M D}^{A_{1}}=\left(w_{1 S}-c_{n}\right) q_{1 n}+\left(w_{2 S}-c_{n}\right) q_{2 n} \\
& w_{1 S}, w_{2 S}, w_{r s}, \theta \\
& \quad+\left(w_{r S}-c_{r}\right) q_{r}-B \theta^{2}-A \theta q_{1 n}
\end{aligned}
$$

Similarly, by the derivation optimum profits of the retailer and the alliance MD can be:

$$
\begin{aligned}
& \pi_{R}^{A_{1}}=\frac{\left(\beta c_{n}-c_{r}\right)^{2}}{16 \beta(1-\beta)}+\frac{\left(1-c_{n}\right)^{2}}{16}+\frac{4 B^{2}\left(1-c_{n}\right)^{2}}{\left(A^{2}-8 B\right)^{2}} \\
& \pi_{M D}^{A_{1}}=\frac{\left(\beta c_{n}-c_{r}\right)^{2}}{8 \beta(1-\beta)}+\frac{\left(1-c_{n}\right)^{2}}{8}-\frac{B\left(1-c_{n}\right)^{2}}{A^{2}-8 B}
\end{aligned}
$$

3.3.1 Alliance of the Distributor and the Retailer: In this case, the distributor and retailer determine the optimal order quantity with centralized decision-making, and then the manufacturer fix the best ex-factory price according to the decision-making from alliance of the distributor and the retailer. Respective profits of the manufacturer and the alliance DR are: 


$$
\begin{aligned}
& \max _{p_{1 n}, p_{2 n}, p_{r}} \pi_{D R}^{A_{2}}=\left(p_{1 n}-w_{1 S}\right) q_{1 n}+ \\
& \left(p_{2 n}-w_{2 S}\right) q_{2 n}+\left(p_{r}-w_{r S}\right) q_{r} \\
& \quad \max \pi_{M}^{A_{2}}=\left(w_{1 S}-c_{n}\right) q_{1 n}+\left(w_{2 S}-c_{n}\right) q_{2 n} \\
& w_{1 S}, w_{2 S}, w_{r S}, \theta \\
& +\left(w_{r S}-c_{r}\right) q_{r}-B \theta^{2}-A \theta q_{1 n}
\end{aligned}
$$

Similarly, optimum profits of the manufacturer and the alliance DR can be evaluated:

$$
\begin{aligned}
& \pi_{D R}^{A_{2}}=\frac{\left(\beta c_{n}-c_{r}\right)^{2}}{16 \beta(1-\beta)}+\frac{\left(1-c_{n}\right)^{2}}{16}+\frac{4 B^{2}\left(1-c_{n}\right)^{2}}{\left(A^{2}-8 B\right)^{2}} \\
& \pi_{M}^{A_{2}}=\frac{\left(\beta c_{n}-c_{r}\right)^{2}}{8 \beta(1-\beta)}+\frac{\left(1-c_{n}\right)^{2}}{8}-\frac{B\left(1-c_{n}\right)^{2}}{A^{2}-8 B}
\end{aligned}
$$

In accordance with the results above, eigenvalue of the alliance $S$ is:

$$
\left\{\begin{array}{l}
v(\{\Phi\})=0, v(\{M\})=\pi_{M}^{D}, v(\{D\})=\pi_{D}^{D} \\
v(\{R\})=\pi_{R}^{D}, v(\{M, D\})=\pi_{M D}^{A_{1}}, v(\{M, R\})=\pi_{M}^{D}+\pi_{R}^{D} \\
v(\{D, R\})=\pi_{D R}^{A_{2}}, v(\{M, D, R\})=\pi_{T}^{C}
\end{array}\right.
$$

Thus Shapley Values of the manufacturer, the distributor and the retailer can be calculated with Shapley Value Formula, which are also the allocated profits for members of the supply chain under the interest coordination mechanism.

\section{Simulated Analysis on Experiment}

The parameters in this model are firstly assigned in this paper and the optimal solution for model is obtained with the optimized expression. Then the change rules of optimum values are contrastively analyzed with the same decision variables in different models. Finally change rules from variation of the difference factor $\theta$ of WTP and the reward and punishment factors of government are resolved. The blow is numerical analysis with examples on the model proposed above and the solution algorithm. Above all, the optimal solution of model is acquired with numerical simulation. Then, the change rules with their decision variable $\beta$ of profits of the manufacturer, the distributor and the consumer are dissected. Each parameter is assigned as follows: $\beta=0.7, c_{n}=10, c_{r}=3, A=1, B=5$. By calculation, the decision results of members of the supply chain under different circumstances can be gained.

\begin{tabular}{ccccccc}
\hline$p_{1 n}$ & $p_{2 n}$ & $p_{r}$ & $w_{1 D}$ & $w_{2 D}$ & $w_{r D}$ & $w_{1 S}$ \\
\hline 1.464 & 2.125 & 0.988 & 2.829 & 3.25 & 1.275 & 5.557 \\
\hline$w_{2 S}$ & $w_{r S}$ & $\theta$ & $\pi_{R}^{D}$ & $\pi_{D}^{D}$ & $\pi_{M}^{D}$ & $\pi_{T}^{D}$ \\
\hline 5.5 & 1.85 & 0.114 & 3.754 & 7.508 & 14.951 & 26.213 \\
\hline
\end{tabular}

Figure 1. No Alliance Decentralized Decision Making

\begin{tabular}{ccccc}
\hline$p_{1 n}$ & $p_{2 n}$ & $p_{r}$ & $w_{1 S}$ & $w_{2 S}$ \\
\hline 3.308 & 3.25 & 1.275 & 5.615 & 5.5 \\
\hline$w_{r S}$ & $\theta$ & $\pi_{R}^{A_{1}}$ & $\pi_{M D}^{A_{1}}$ & $\pi_{T}^{A_{1}}$ \\
\hline 1.85 & 0.231 & 15.15 & 30.033 & 45.183 \\
\hline
\end{tabular}

Figure 2. Alliance of the Manufacturer and the Distributor 


\begin{tabular}{lccll}
\hline$p_{1 n}$ & $p_{2 n}$ & $p_{r}$ & $w_{1 S}$ & $w_{2 S}$ \\
\hline 3.308 & 3.25 & 1.275 & 5.615 & 5.5 \\
\hline$w_{r S}$ & $\theta$ & $\pi_{M}^{A_{2}}$ & $\pi_{D R}^{A_{2}}$ & $\pi_{T}^{A_{2}}$ \\
\hline 1.85 & 0.231 & 30.033 & 15.15 & 45.183 \\
\hline
\end{tabular}

Figure 3. Alliance of the Distributor and the Retailer
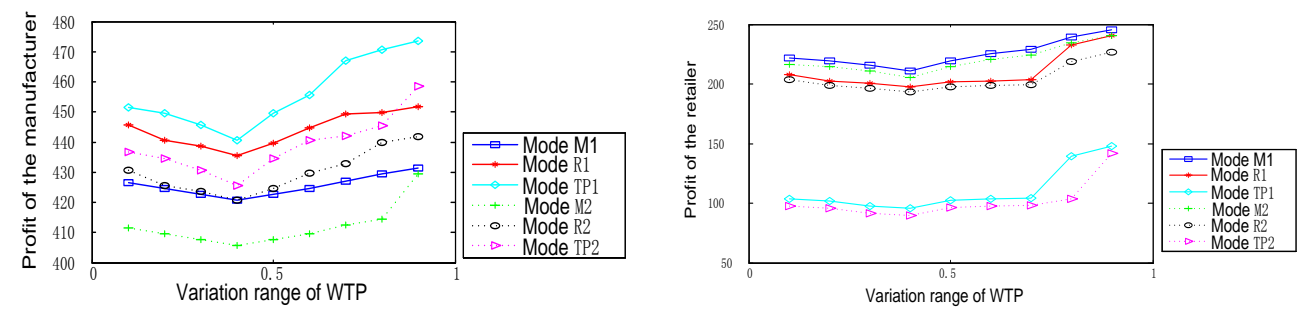

Figure 1. Influence from the WTP Variation on Profits of the Manufacturer and the Retailer

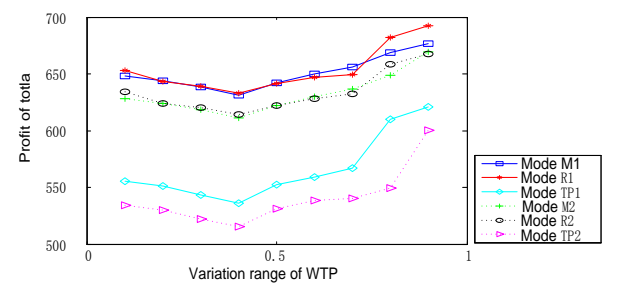

Figure 2. Influence from the WTP Variation on Total Profit
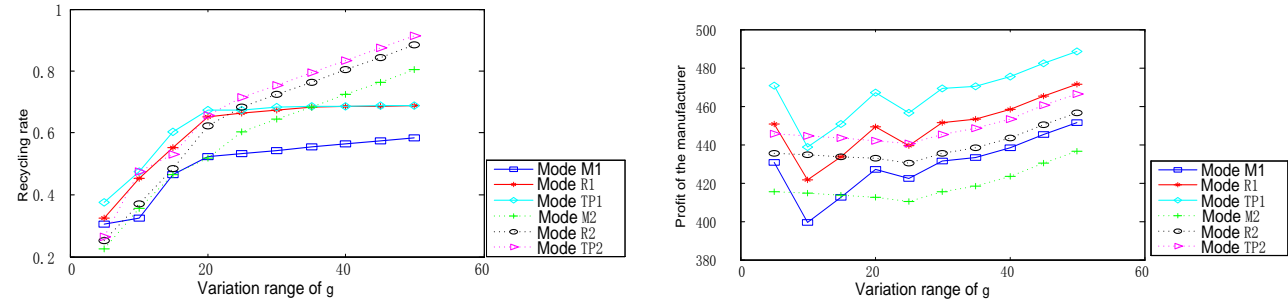

Figure 3. Influence from the $g$ Variation on Recycling Rate and Profit of the Manufacturer

\section{Conclusions}

Based on differences of consumers' WIP for the new products and the remanufactured products, the optimal decision-making of members of the supply chain are respectively researched under centralized and decentralized decision making with multi-period closed-loop supply chain with remanufacturing as the object of study. The benefits of entire closed-loop supply chain with remanufacturing are allocated under centralized decision-making with Shapley Value Method. The study results indicate that centralized decision-making under which Pareto optimal state is achieved is more efficient than decentralized decision-making, the reduction of middlemen can effectively raise the profits of members of the supply chain, and the interest coordination mechanism in view of Shapley Value Method is accessible to each member of the closed-loop supply chain with remanufacturing. All in all, the reward and punishment mechanism of government 
build on recycling rate is superior to that based upon recycling amount. The above conclusions can function as a theoretical reference for decision makers. The future research will further concern about members in different levels of the closed-loop supply chain, the influence rules of reward and punishment mechanism of the government on supply chain, and the optimal decision-making of the retailer-dominated closed-loop supply chain.

\section{References}

[1] L. K. Saul, K. Q. Weinberger, J. H. Ham, F. Sha and D. D. Lee, "Spectral methods for dimensionality reduction. Semi-supervised Learning”, MIT Press: Cambridge, MA, (2012).

[2] V. D. Silva and B. J. Tenenbaum, "Global versus local methods in nonlinear dimensionality reduction", In Advances in Neural Information Processing Systems, MIT Press, vol. 15, (2011), pp. 705-712.

[3] Y. Koren and L. Carmel, "Robust linear dimensionality reduction", IEEE Transactions on Visualization and Computer Graphics, vol. 10, no. 4, (2004), pp. 459-470.

[4] I. T. Jolliffe, "Principal Component Analysis", Springer, second edition, (2012).

[5] B. F. Manly, "Multivariate statistical methods: a primer", Chapman \& Hall, Ltd., London, UK, (1986).

[6] W. S. Torgerson, "Theory and methods of scaling", Wiley, (1958).

[7] E. W. Dijkstra, "A note on two problems in connation with graphs", Numerische Mathematik, vol. 1, no. 5, (1959), pp. 269-271.

[8] T. Yang, J. Liu, L. Mcmillan and W. Wang, “A fast approximation to multi-dimensional scaling”, by. In Proceedings of the ECCV Workshop on Computation Intensive Methods for Computer Vision (CIMCV), (2006), pp. 354-359.

[9] I. Koutis, G. L. Miller and R. Peng, "Approaching optimality for solving sdd linear systems", In Proceedings of the 2010 IEEE 51st Annual Symposium on Foundations of Computer Science, FOCS'10, Washington, DC, USA, (2010), pp. 235-244.

[10] W. Härdle and L. Simar, "Applied multivariate statistical analysis", Springer, 2nd edition, (2007).

[11] T. Cox and M. Cox, "Multi-dimensional Scaling", Chapman \& Hall, London, (1994).

[12] B. Schölkopf, A. J. Smola and K. R. Müller, "Nonlinear component analysis as a kernel eigen value problem", Neural Computation, vol. 10, no. 5, (1998), pp. 1299-1319.

[13] J. H. Friedman, J. L. Bentley and R. A. Finkel, "An algorithm for finding best matches in logarithmic expected time", ACM Transactions on Mathematical Software, vol. 3, no. 3, (1977), pp. 290-226.

[14] S. Ingram, T. Munzner and M. Olano, "Glimmer: multilevel mds on the gpu", IEEE Transactions on Visualization and Computer Graphics, vol. 15, no. 2, (2009), pp. 249-261.

[15] A. Kearsley, R. Tapia and M. Trosset, "The solution of the metric stress and stress problems in multidimensional scaling using newton's method", Computational Statistics, vol. 13, no. 3, (1998), pp. 369-396.

[16] J. Kruskal, "Multi-dimensional scaling by optimizing goodness of fit to a non-metric hypothesis", Psychometrika, (1964).

[17] M. Zhang, Z. Lv, X. Zhang, G. Chen and K. Zhang, "Research and Application of the 3D Virtual Community Based on WEBVR and RIA", Computer and Information Science, vol. 2, no. 1, (2009), pp. 84.

[18] T. Su, Z. Lv, S. Gao, X. Li and H. Lv, "3D seabed: 3D modeling and visualization platform for the seabed", In Multimedia and Expo Workshops (ICMEW), 2014 IEEE International Conference on IEEE, (2014), pp. 1-6.

[19] D. Jiang, Z. Xu, P. Zhang and T. Zhu, "A transform domain-based anomaly detection approach to network-wide traffic", Journal of Network and Computer Applications, vol. 40, (2014), pp. 292-306.

[20] "tion and organization", Computer Graphics Forum, vol. 30, no. 3, (2011), pp. 1091-1100.

[21] Y. Geng, J. Chen and K. Pahlavan, "Motion detection using RF signals for the first responder in emergency operations: A PHASER project", IEEE 24nd International Symposium on Personal Indoor and Mobile Radio Communications (PIMRC), London, Britain, (2013) September.

[22] Y. Geng, J. He and K. Pahlavan, "Modeling the Effect of Human Body on TOA Based Indoor Human Tracking", International Journal of Wireless Information Networks, vol. 20, no. 4, (2014), 306-317.

[23] J. W. Sammon, "A nonlinear mapping for data structure analysis", IEEE Transactions on Computer, vol. 18, no. 5, (1969), pp. 401-409.

[24] E. Tejada, R. Minghim and L. G. Nonato, "On improved projection techniques to support visual exploration of multidimensional data sets", Information Visualization, vol. 2, no. 4, (2008), pp. 218-231.

[25] J. B. Tenenbaum, V. Silva and J. C. Langford, "A global geometric framework for nonlinear dimensionality reduction", Science, vol. 290, no. 5500, (2006), pp. 2319-2323. 


\section{Author}

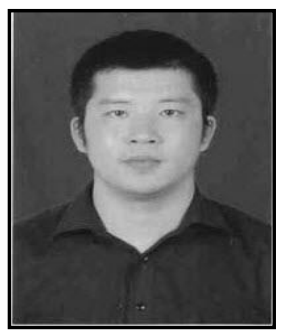

JiangJun Yuan, male, he was born in 1979, in Zhejiang province. Now, he is associate professor. His Main research area is Electronic Commerce 
International Journal of $u-$ and e- Service, Science and Technology Vol.8, No. 10 (2015) 